\title{
Systematic review: agreement between the latent tuberculosis screening tests among patients with rheumatic diseases
}

\author{
Junhee $\mathrm{Pyo}^{1}$, Soo-Kyung $\mathrm{Cho}^{2}$, Dam Kim² ${ }^{2}$ and Yoon-Kyoung Sung ${ }^{2}$
}

\author{
${ }^{1}$ WHO Collaborating Centre \\ for Pharmaceutical Policy and \\ Regulation, Department of \\ Pharmaceutical Science, Utrecht \\ University, Utrecht, the Netherlands; \\ ${ }^{2}$ Department of Rheumatology, \\ Hanyang University Hospital for \\ Rheumatic Diseases, Seoul, Korea
}

Received: July 7, 2016

Revised : November 12, 2016

Accepted: November 16, 2016

Correspondence to

Yoon-Kyoung Sung, M.D.

Department of Rheumatology, Hanyang University Hospital

for Rheumatic Diseases, 222-1

Wangsimni-ro, Seongdong-gu,

Seoul 04763, Korea

Tel: +82-2-2290-9207

Fax: +82-2-2298-8231

E-mail: sungyk@hanyang.ac.kr
Background/Aims: To estimate the level of agreement and positivity rates of latent tuberculosis infection (LTBI) tests prior to the use of tumor necrosis factor (TNF) inhibitors in relation to underlying rheumatic diseases and endemic tuberculosis levels.

Methods: The Ovid-Medline, Embase, and Cochrane Libraries were searched for articles before October 2013 involving LTBI screening in rheumatic patients, including rheumatoid arthritis (RA), ankylosing spondylitis (AS), juvenile idiopathic arthritis (JIA), and psoriatic arthritis.

Results: In pooled analyses, 5,224 rheumatic patients had undergone both a tuberculin skin test (TST) and an interferon-gamma release assay (IGRA) before TNF inhibitors use. The positivity of TST, QuantiFERON-TB Gold In Tube (QFTGIT), and T-SPOT.TB (T-SPOT) tests were estimated to be $29 \%, 17 \%$, and $18 \%$, respectively. The agreement percentage between the TST and QFT-GIT, and between the TST and T-SPOT were 73\% and 75\%. Populations from low-to-moderate endemic TB presented with slightly less agreement (71\% between TST and QFT-GIT, and 74\% between TST and T-SPOT) than patients from high endemic countries (73\% between TST and QFT-GIT, and 81\% between TST and T-SPOT). By underlying disease stratification, a lower level of agreement between TST and QFT-GIT was found among AS (64\%) than among JIA (77\%) and RA patients (73\%). Conclusions: We reaffirm the current evidence for accuracy of LTBI test done by TST and IGRA among rheumatic patients is inconsistent. Our stratified analysis suggests different screening strategies might be needed in clinical settings considering the endemic status in the patient's country of origin and the precise nature of underlying diseases.

Keywords: Latent tuberculosis; Rheumatic diseases; Interferon-gamma release tests; Tuberculin test; Tumor necrosis factor-alpha

\section{INTRODUCTION}

Tuberculosis (TB) remains a major concern and is extremely prevalent in patients with rheumatic diseases due to their immune dysregulation and the immuno- suppressive agents used in their treatment [1]. Subsequent to the introduction of biologic agents, the reactivation of latent tuberculosis infection(LTBI) and new TB infections both increase. Therefore, it is recommended that all patients who are candidates for treatment 
with tumor necrosis factor (TNF) inhibitors should be screened for Mycobacterium tuberculosis infection before treatment is initiated $[2,3]$. Recently, in 2012, the American College of Rheumatology (ACR) recommended the use of a tuberculin skin test (TST) or an interferon-gamma release assay (IGRA) to identify LTBI in rheumatoid arthritis (RA) patients who are being considered for biologic agent therapy [4].

Screening tests such as the TST and IGRA for LTBI, are commercially available; these would include the U.S. Food and Drug Administration (FDA)-approved QuantiFERON-TB Gold In Tube (QFT-GIT; ELISA, Cellestis Ltd., Carnegie, Australia) or the T-SPOT.TB (T-SPOT; Elispot, Oxford Immunotec Inc., Oxford, UK). They have been tested for accuracy, but the agreement levels vary across all studies [5-24]. Although several studies have evaluated diagnostic accuracy and agreement across LTBI screening tests implemented before starting TNF inhibitors with patients with rheumatic diseases, there has been controversy between the individual studies and between countries.

In this study, we conducted a meta-analysis of the level of agreement and positivity rates of LTBI screening tests prior to the use of TNF inhibitors according to the underlying rheumatic disease and the endemic TB status of each country. We then calculated the proportion of patients with rheumatic diseases targeted for treatment for LTBI before starting biologic agents according to each screening strategy and according to the endemic TB status of their countries of origin.

\section{METHODS}

\section{Literature search strategy}

A computerized search of the Ovid-Medline, Embase, and Cochrane databases was conducted to find relevant studies published prior to October, 2013. We did not restrict the start date. The following search terms were used: (latent tuberculosis) AND [(rheumatoid AND arthritis) OR (ankylosing AND spondylitis) OR (juvenile AND idiopathic AND arthritis) OR (psoriatic AND arthritis)]. Our search was restricted to human subjects and to articles written in English. We also screened the bibliographies of selected papers to find other eligible articles.

\section{Inclusion criteria}

Studies were eligible for inclusion if patients with rheumatic diseases were screened for the detection of LTBI prior to the use of TNF inhibitors. Studies that satisfied all of the following criteria were included (1) population: patients with rheumatic diseases such as RA, psoriatic arthritis (PsA), ankylosing spondylitis (AS), and juvenile idiopathic arthritis (JIA) were included. All patients enrolled in the studies met the ACR criteria for the classification of rheumatic diseases; (2) intervention: LTBI screenings using either TST or one IGRA (QFT-GIT or T-SPOT) had been conducted, mostly before using TNF inhibitors (more than $90 \%$ of the population should not have previously used TNF inhibitors); (3) study designs: all observational studies (retrospective or prospective) and clinical trials; and (4) outcomes: results reported in sufficient detail to obtain a positive rate of LTBI and an agreement percentage between TST and one IGRA.

\section{Exclusion criteria}

The exclusion criteria were as follows: (1) case reports, case series, review articles, editorials, letters, comments, and conference abstracts; (2) studies with insufficient data to calculate a positive rate or agreement percentage between TST and one IGRA; (3) studies that used a nonFDA approved LTBI screening test; (4) studies including more than $10 \%$ of patients on current or previous TNF inhibitors. However, if the studies included a minor proportion of patients who were currently using or had previously used TNF inhibitors, which was less than $10 \%$ of the total population, these studies were included; (5) studies out of the field of interest; and (6) studies that overlapped with other studies due to patient overlap. Two reviewers independently reviewed the literature using a standardized protocol, and disagreements were resolved by a meeting where a consensus was established.

\section{Data extraction}

We extracted the data available for a meta-analysis from the studies included as follows: (1) study characteristics (authors, year of publication, location and period of study, population size, and study design); (2) demographics and clinical characteristics of the patients (age, sex, disease duration of rheumatic disease, concomitant medications, Bacille Calmette-Guerin [BCG] vaccination status, specific rheumatic disease type, and coun- 
try setting); and (3) Outcome characteristics: number of positive cases and number of total cases collected to calculate the positive rates of LTBI as measured by TST and IGRA. A number of pairs of TST results and IGRA results, including TST+/IGRA+, TST+/IGRA-, TST-/ IGRA+, and TST-/IGRA- were extracted to estimate the agreement percentages between the two screening tests. Two reviewers (J.P. and Y.K.S.) extracted the data from the studies by consensus.

\section{Data synthesis and statistical analysis}

To explore the discrepancies in the prevalence as measured by the TST and the IGRAs, a pooled positive rate and an agreement percentage across the LTBI screening tests before TNF inhibitor use were adopted as the meta-meters of our meta-analysis. The positive rate of a test is the proportion of positive cases to the total number of the population screened. In addition, we pooled the data for the agreement percentage (\%), which was adopted as an indicator of the agreement level across the diagnostic tests, as the agreement index for this analysis. The agreement percentage (\%) is the proportion of the concordance cases to the total number of concordance and discordance cases between the TST and one IGRA.

We obtained the pooled positive rates and the agreement percentage calculated from all the studies. We then performed two sets of subgroup analyses stratified by the endemic TB rating of the region and the specific rheumatic disease. First, we stratified the studies and synthesized the outcomes of interest according to the endemic TB regions as based on the country settings. This was done to explore the differences across countries with various TB endemic ratings. According to the TB prevalence per 100,000 population from the 2011 World Health Organization (WHO) report, we classified the studies into two categories: studies conducted in low-to-moderate endemic TB regions (less than 20/100,000), and high endemic TB regions (more than 20/100,000). Next, we stratified the studies according to the specific rheumatic diseases (RA, AS, JIA) provided a specific rheumatic disease was reported as a subset with a separate outcome analysis in the article. DerSimonian-Laird random-effect models were constructed to synthesize the pooled positive rate and the agreement percentage with 95\% confidence intervals (CIs) in order to consider potential heterogeneity across studies.
This methodological approach was based on an inverse variance technique to calculate the weights and pooled outcomes with $95 \%$ CIs. To investigate the heterogeneity, we calculated the Cochran's Q test and the $I^{2}$ statistics, which were considered significant if the $I^{2}$ statistics were greater than $50 \%$, and the $p$ value of the Cochran's $\mathrm{Q}$ test was less than 0.10 . To assess publication bias, we plotted funnel plots and conducted Begg's test to further detect asymmetry.

In addition, we estimated the number and proportion of patients with rheumatic diseases targeted for LTBI treatment according to each screening strategy across the TB endemic regions. First, we constructed a $2 \times 2$ diagnostic table consisting of patient distribution in the literature included to calculate the number of patients and proportion of positive results among the total population across the TST and the IGRAs. We assumed that patients who had positive screening results according to each strategy were all supposed to receive LTBI treatment. Four screening strategies were used to determine further prophylactic treatment: TST only positive, IGRA only positive, both TST and IGRA positive, and either TST or IGRA positive were all examined. The $\mathrm{R}$ version 3.0.1 (https://www.r-statistics.com/2013/05/r-3-0-1-is-released/) package was used for analysis with the metafor command.

This systematic review was conducted according to the Preferred Reporting Items for Systematic Revise and Meta-Analyses (PRISMA) statement.

\section{RESULTS}

\section{Literature search}

Our literature search process is illustrated in Fig. 1. After removing duplicates, 280 articles were screened for eligibility in the Ovid-Medline, Embase, and Cochrane databases. Of these, 253 articles were excluded after a review of the titles and abstracts: 171 were reviews/letters/editorials/abstracts; seven were case reports/series; six studies used a non-FDA approved LTBI screening test; three studies included patients on current or previous TNF inhibitors; 10 studies had no comparisons across LTBI screening tests; 10 studies used LTBI tests for non-rheumatic diseases (e.g., other autoimmune diseases, human immunodeficiency virus [HIV]); and 
46 studies were out of the field of interest (e.g., safety/ efficacy studies of TB prophylaxis or studies evaluating TB incidence).

The full texts of the remaining 27 articles were retrieved. The following nine articles were then excluded after a review of the full texts: three studies included patients on current or previous TNF inhibitors; three studies had no comparisons across the LTBI screening tests; one study included insufficient data to yield our meta-meters of interest; one study did not separate the data for patients with rheumatic diseases from that for other autoimmune diseases; and one study was out of the field of interest for our research. On searching the bibliographies of the articles included two additional eligible studies were included $[18,19]$. Finally, a total of

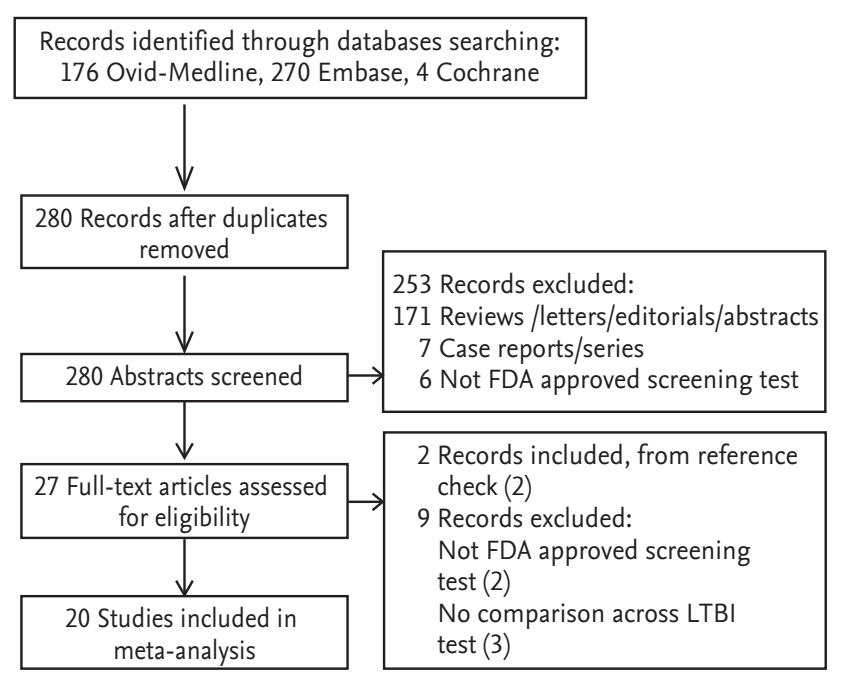

Figure 1. Flow diagram for the literature search. FDA, U.S. Food and Drug Administration; LTBI, latent tuberculosis infection.
20 studies were used for the qualitative and quantitative review [1-20].

\section{Study characteristics}

The basic characteristics of the 20 studies are summarized in the Supplementary Table 1. The author, publication year, disease population, number of patients, age, female proportion, concomitant medications, previous BCG vaccination, disease duration, country setting, comparison groups with cut-off information, and summarized positive rate and agreement level of each study are presented in detail, and stratified according to the TB endemic regions (Supplementary Table 1). Nine studies, with a total of 1,359 patients, were conducted in high endemic TB areas (Brazil, China, Peru, Poland, Korea, Taiwan, and Turkey); 10 studies with a total of 1,493 patients were in low-to-moderate TB endemic settings (Canada, Czech, France, Greece, Ireland, Israel, and Spain); and one study of 2,282 patients was conducted in a global setting across all regions (Asia, North/Latin America, and Europe). In this review, the total population was 5,224, which included 3,026 clearly defined RA patients (57.9\%), 1,009 AS patients (19.3\%), 559 PsA patients (10.7\%), 141 JIA patients (2.7\%), and 489 others (9.3\%), including the unknown disease population. A TST positive test was conducted among 5,051 patients, QFT-GIT among 4,799 patients, and T-SPOT among 846 patients. The agreement level between TST and QFT-GIT was compared among 4,830 patients and between TST and T-SPOT among 864 patients.

\section{Positive rates for the LTBI screening tests}

The pooled positive rates among all the 5,051 patients with rheumatic diseases across the LTBI screening tests

Table 1. Estimated number of patients according to screening strategies for latent tuberculosis infection treatment

\begin{tabular}{lccc}
\hline Variable & Total & High endemic & Low to moderate endemic \\
\hline TST only positive & $736(32.8)$ & $502(34.8)$ & $234(29.3)$ \\
IGRA only positive & $507(22.6)$ & $390(27.0)$ & $117(14.7)$ \\
TST and IGRA positive & $300(13.4)$ & $243(16.8)$ & $57(7.1)$ \\
TST or IGRA positive & $943(42.0)$ & $649(55.0)$ & $294(36.8)$ \\
Total no. of patients across all strategies & 2,241 & 1,443 & 798 \\
\hline
\end{tabular}

Values are presented as number (\%). This table represents the estimated number of patients targeted for latent tuberculosis infection treatment according to each screening strategy as a subset of our literature review.

TST, tuberculosis skin test; IGRA, interferon-gamma release assay. 
before the use of TNF inhibitors are summarized in Fig. 2A. The overall positive rates for TST (> $5 \mathrm{~mm}$ ), QFTGIT, and T-SPOT among all the patients included under the random-effect modeling were estimated as $29 \%$ (95\% CI, 24 to 34 ), $17 \%$ (95\% CI, 11 to 23 ), and 18\% (95\% CI, 11 to 26), respectively. A greater positive diagnosis of LTBI was found on the TST test than on the QFT-GIT or T-SPOT.

In a stratified analysis according to TB endemic region (Fig. 2A), all patients with rheumatic diseases $(\mathrm{n}=$ 1,322) in the low-to-moderate endemic setting presented positive rates by TST, QFT-GIT, and T-SPOT of $33 \%$ ( $95 \%$ CI, 25 to 41 ), $10 \%$ (95\% CI, 6 to 14), and $20 \%$ (95\% CI, 15 to 25$)$, respectively. Among all patients with rheumatic

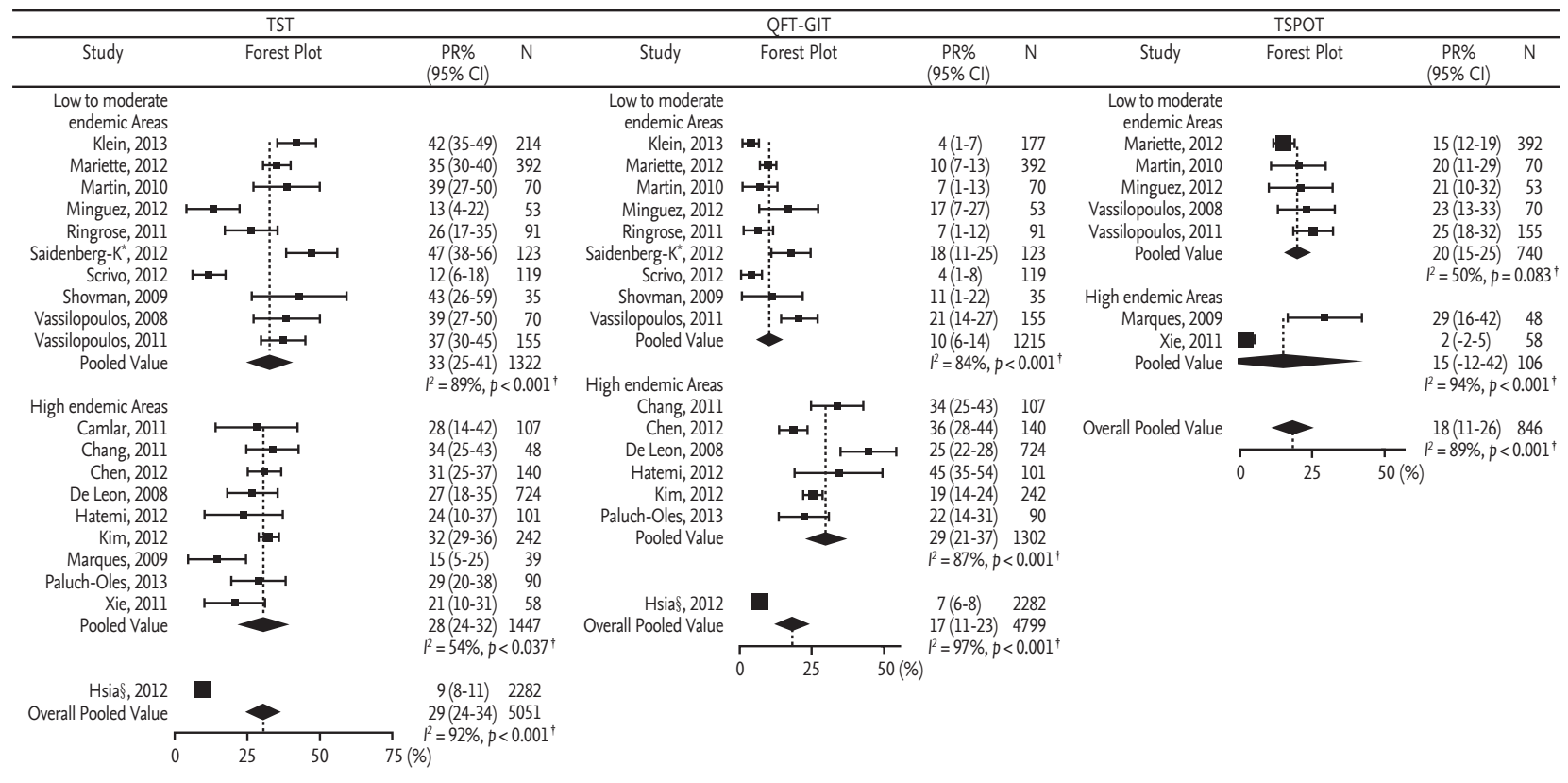

"Saidenberg-Kermanac'h et al's study

${ }^{\dagger} p$-value of Q-test.

A

$\S$ Hsia et al's study was a multi-nation study and included low to moderate endemic and high endemic areas.

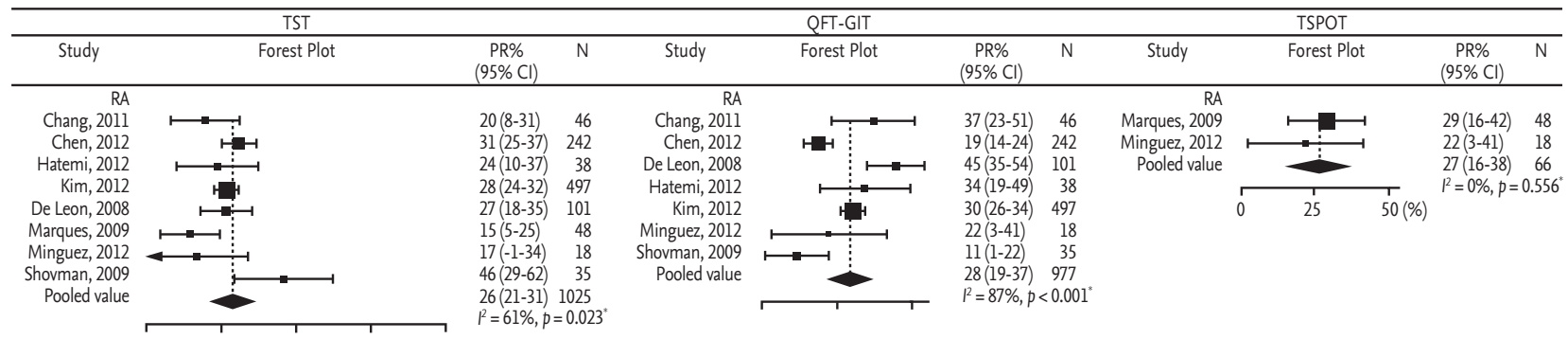

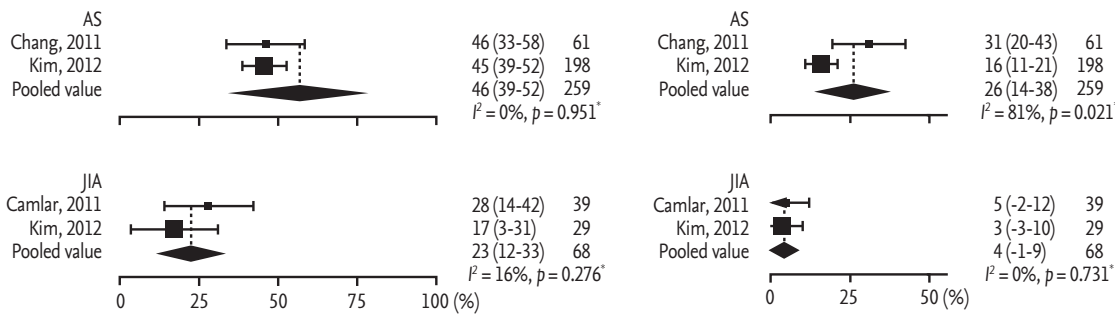

B

" $p$-value of Q-test.

Figure 2. Pooled analysis of the positive rate (PR\%) of latent tuberculosis infection screening tests (A) by tuberculosis endemic area and (B) by disease type. TST, tuberculin skin test; QFT-GIT, QuantiFERON-TB Gold In Tube; CI, confidence interval; TSPOT, T-SPOT.TB test; N, number; RA, rheumatoid arthritis; AS, ankylosing spondylitis; JIA, juvenile idiopathic arthritis. 
diseases $(n=1,447)$ from the high endemic settings, the positive rates by TST, QFT-GIT, and T-SPOT were $28 \%$ (95\% CI, 24 to 32 ), $29 \%$ ( $95 \%$ CI, 21 to 37 ), and $15 \%$ ( $95 \%$ CI, -12 to 42 ), respectively. In this analysis, substantial heterogeneity was found, evidenced by an $I^{2}$ value greater than $50 \%$ and a $p$ value $<0.10$ for the $Q$ test.

In the subgroup analysis according to the underlying rheumatic diseases (Fig. 2B), the RA population ( $\mathrm{n}=$ 1,025) presented consistent positive rates by TST, QFTGIT, and T-SPOT of $26 \%$ to $28 \%$. Among the AS and JIA patients, the positivity rates were not consistent according to the LTBI screening tests. Positivity measured by TST was significantly larger than that by QFTGIT among the AS and JIA populations. Heterogeneity across studies seemed to be attenuated when analyzed according to the specific underlying disease type, even though significant heterogeneity still existed among RA populations for both the TST and QFT-GIT positive rates, and among AS populations for the QFT-GIT positive rates $(\mathrm{Q}$ test; $p=0.023, p<0.0001$, and $p=0.021$, respectively). However, we cannot exclude the possibility that the nonheterogeneity was potentially driven by the small number of studies among the subgroups.

No statistically significant publication or reporting bias existed in the pooled positive rates of TST, QFTGIT, and T-SPOT, as demonstrated by the nonsignificant Begg's test results (Fig. 3).

\section{Percentage agreement in the LTBI screening tests}

The pooled agreement percentage among all the 4,830 patients with rheumatic diseases across the LTBI screening tests is summarized in Fig. 4. The overall agreement percentage between TST ( $>5 \mathrm{~mm}$ ) and QFT-GIT, and between TST (> $5 \mathrm{~mm}$ ) and T-SPOT among all patients resulted in $73 \%$ (95\% CI, 68 to 78 ) and $75 \%$ (95\% CI, 70 to 80), respectively (Fig. 4A).

Even though the differences in the agreement percentage by TB endemic stratification were not substantial (Fig. 4A), the agreement level in the low to moderate endemic areas appears to be numerically lower (71\% for TST vs. QFT-GIT, 74\% for TST vs. T-SPOT) than that of the high endemic areas (73\% for TST vs. QFT-GIT, 81\% for TST vs. T-SPOT). In addition, there were significant heterogeneities across all the stratified analyses.

Subgroup analyses by the underlying diseases RA, AS, and JIA showed that the agreement percentage between the TST and the QFT-GIT was lower among the AS patients (64\%; $95 \%$ CI, $58 \%$ to $70 \%$ ) than among the RA patients (73\%; $95 \%$ CI, $69 \%$ to $78 \%$ ) and the JIA patients (77\%; 95\% CI, $58 \%$ to $96 \%$ ). The subgroup analysis, measuring the agreement level between TST and T-SPOT, was available only for the RA population, which presented as $86 \%$ (95\% CI; 77 to 95). Heterogeneity seems to be attenuated by the subgroup analysis; however, we cannot exclude the possibility that the smaller number of studies resulted in the nonheterogeneity.

No statistically significant publication or reporting bias existed for the pooled agreement percentage between the TST and QFT-GIT, or between the TST and T-SPOT, a result which was derived from all the studies included, as demonstrated by the nonsignificant Begg's test results (Fig. 5).

\section{Estimated number and proportion of patients for LTBI treatment}

In high endemic regions, $34.8 \%$ of patients are targeted for treatment for LTBI according to a TST-only positive strategy, $27.0 \%$ of patients per a IGRA-only positive strategy, $16.8 \%$ of patients per a both TST and IGRA positive strategy, while $55.5 \%$ of patients were targeted per an either TST or IGRA positive strategy as needing LTBI treatment (Table 1). In low to moderate endemic regions, $29.3 \%, 14.7 \%, 7.1 \%$, and $36.8 \%$ of patients needed LTBI treatment according to the screening strategies described above The screening strategy that resulted in the largest proportion of patients requiring LTBI prophylaxis was the either TST or IGRA positive, followed by the TST-only positive, the IGRA-only positive, and last the both TST and IGRA positive outcome.

\section{DISCUSSION}

We reaffirm that the current evidence for the accuracy of the LTBI screening test as done by the TST and IGRA among patients with rheumatic diseases is inconsistent. We also found that among all patients with rheumatic diseases there were more positive diagnoses by TST than were obtained by screenings done with either of the IGRA tests. However, in this meta-analysis, we detected a numerical indication that populations originating from low-to-moderate endemic TB regions present- 

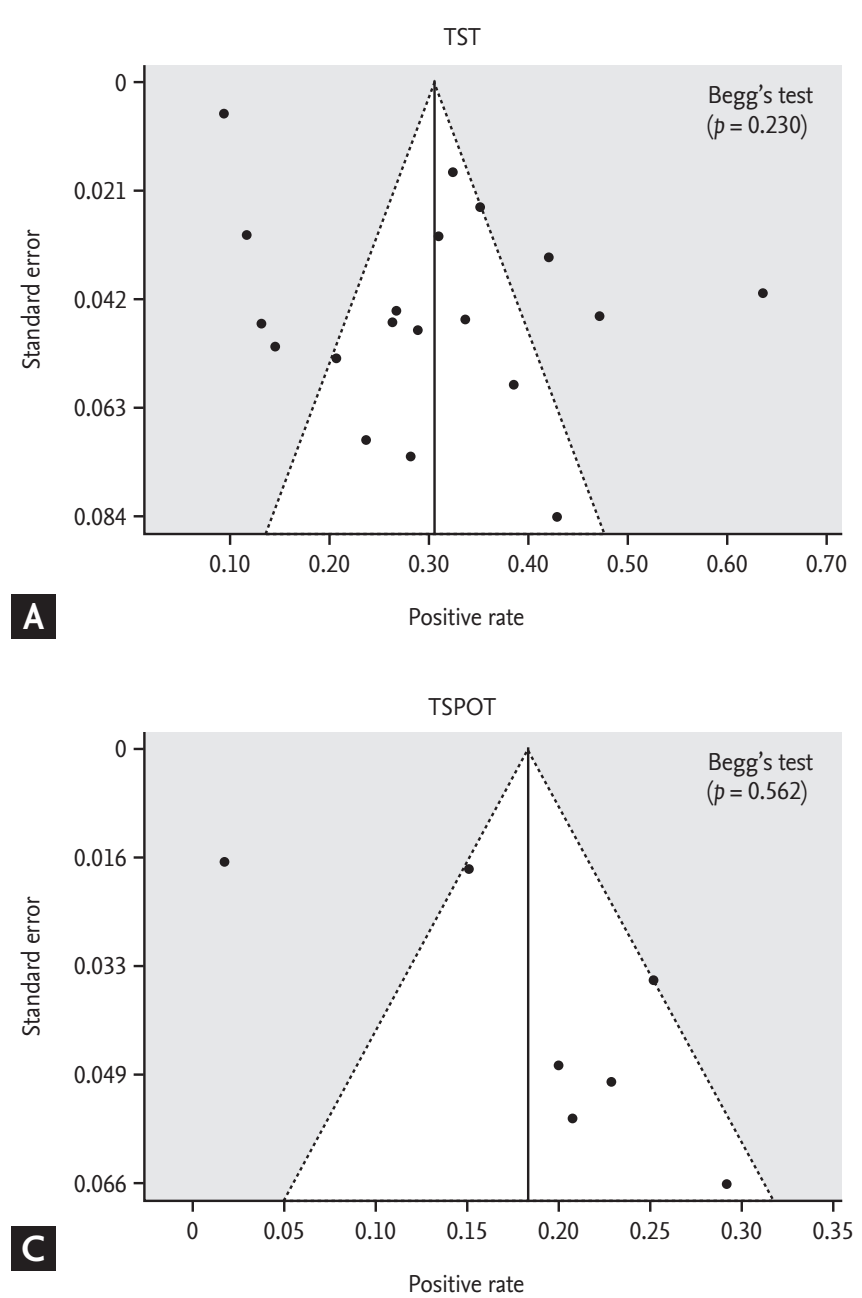

ed with slightly more disagreement in accuracy, mainly due to the lower positive rates from TST being closer to the rates obtained by IGRAs, than in patients from the high endemic TB regions. When stratified according to underlying disease, a lower level of agreement between TST and IGRAs was found among the AS patients than among the RA and JIA patients. However, we have to interpret this with caution since it was not statistically significant.

The lack of a gold standard for diagnosing LTBI makes an assessment of the diagnostic performance of TST and the IGRAs contentious [25]. The TST is a long-established method widely used to identify LTBI due to its simplicity and efficiency, although it has several inherent drawbacks, such as a cross-reaction with the BCG vaccination and other environmental mycobacteria infections resulting in variability in interpreting the results $[26,27]$. Testing for LTBI using risk factors and

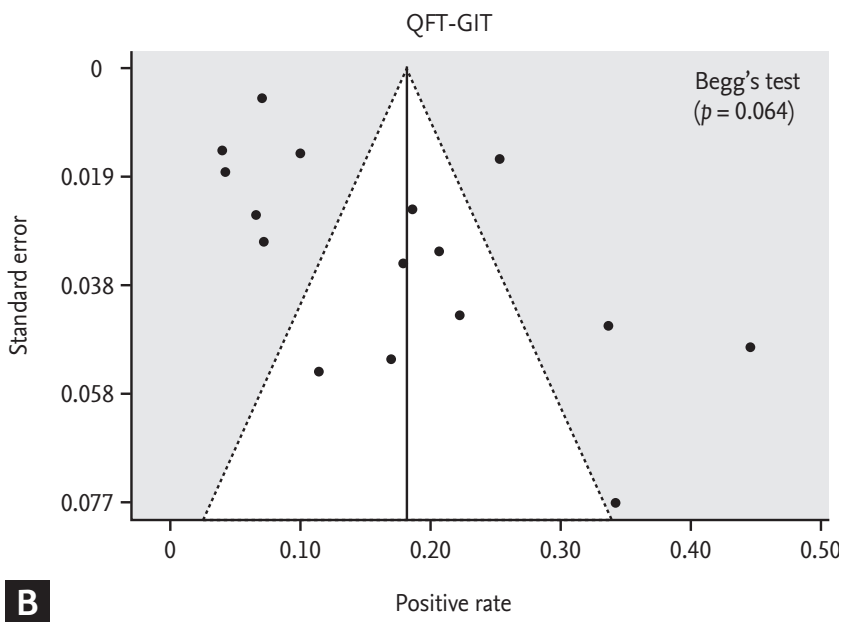

Figure 3. Assessment of publication bias through funnel plots for positive rates. (A) Tuberculin skin test (TST), (B) QuantiFERON TB-Gold In-Tube test (QFT-GIT, Cellestis Ltd.), and (C) T-SPOT.TB (TSPOT, Oxford Immunotec Inc.).

TST has been shown to reduce the incidence of reactivation of tuberculosis in patients taking TNF inhibitors. IGRAs have shown excellent results for screening after exposure to tuberculosis. However, their ability to detect LTBI in patients with rheumatic diseases is not yet proven.

Recommendations for screening for LTBI in current guidelines vary on the subject of replacing TST with IGRAs or of utilizing both tests [28]. In general, the current international guidelines recommend TST first and IGRA in cases of suspected false-negative TST in patients at risk for $M$. tuberculosis exposure, or if a history of previous BCG vaccination is present, but in Switzerland, TST is no longer recommended, and IGRA is the only test advised [29]. In addition, it has been shown that serial TST together with IGRA may be useful to identify a false-negative response in cases of LTBI and new TB infections during long-term TNF inhibitor use, es- 


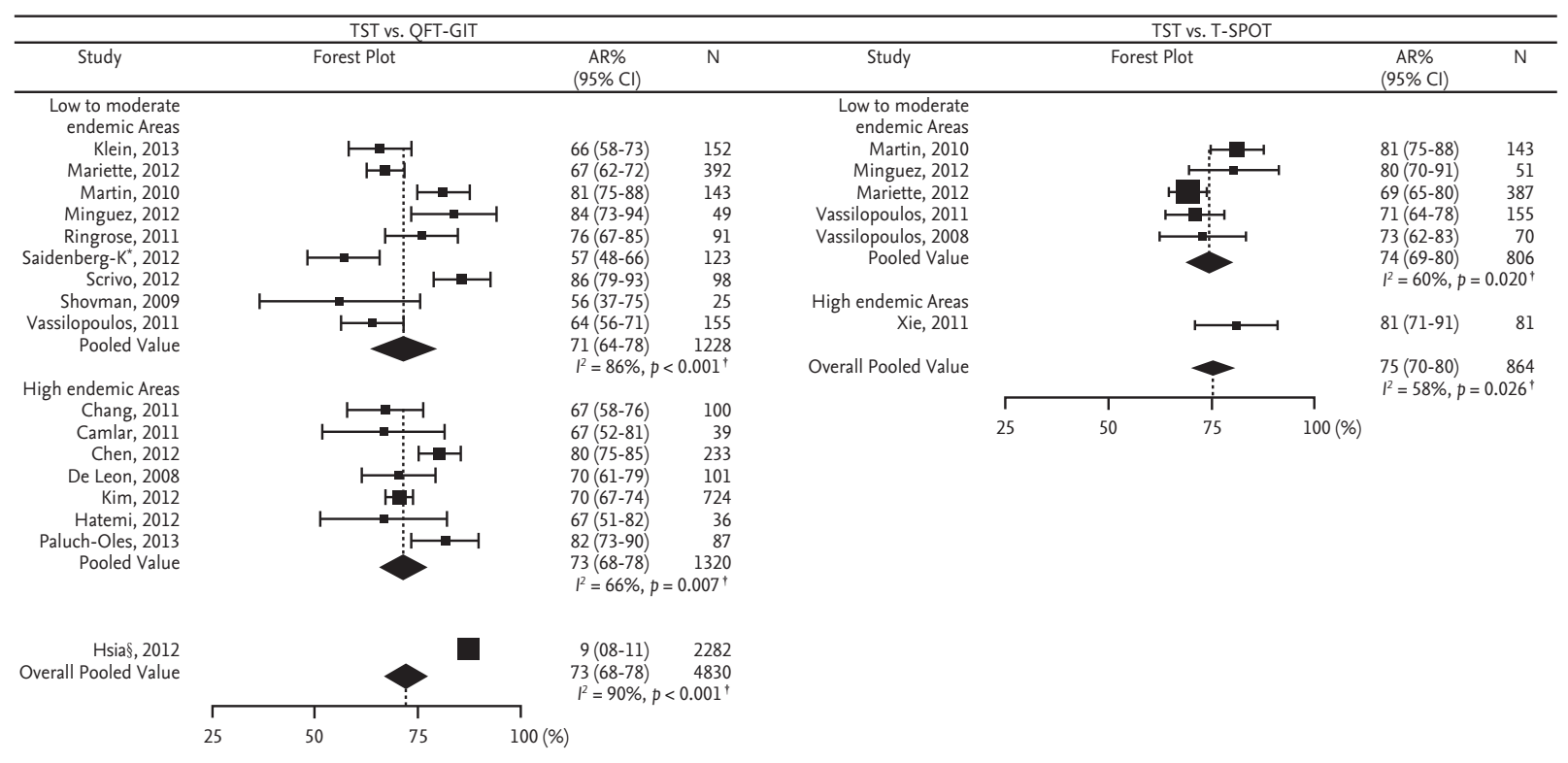

* Saidenberg-Kermanac'h et al's study

$p$-value of Q-test.

p-value of Q-test.
\$ Hsia et al's study was a multi-nation study and included low to moderate endemic and high endemic areas.

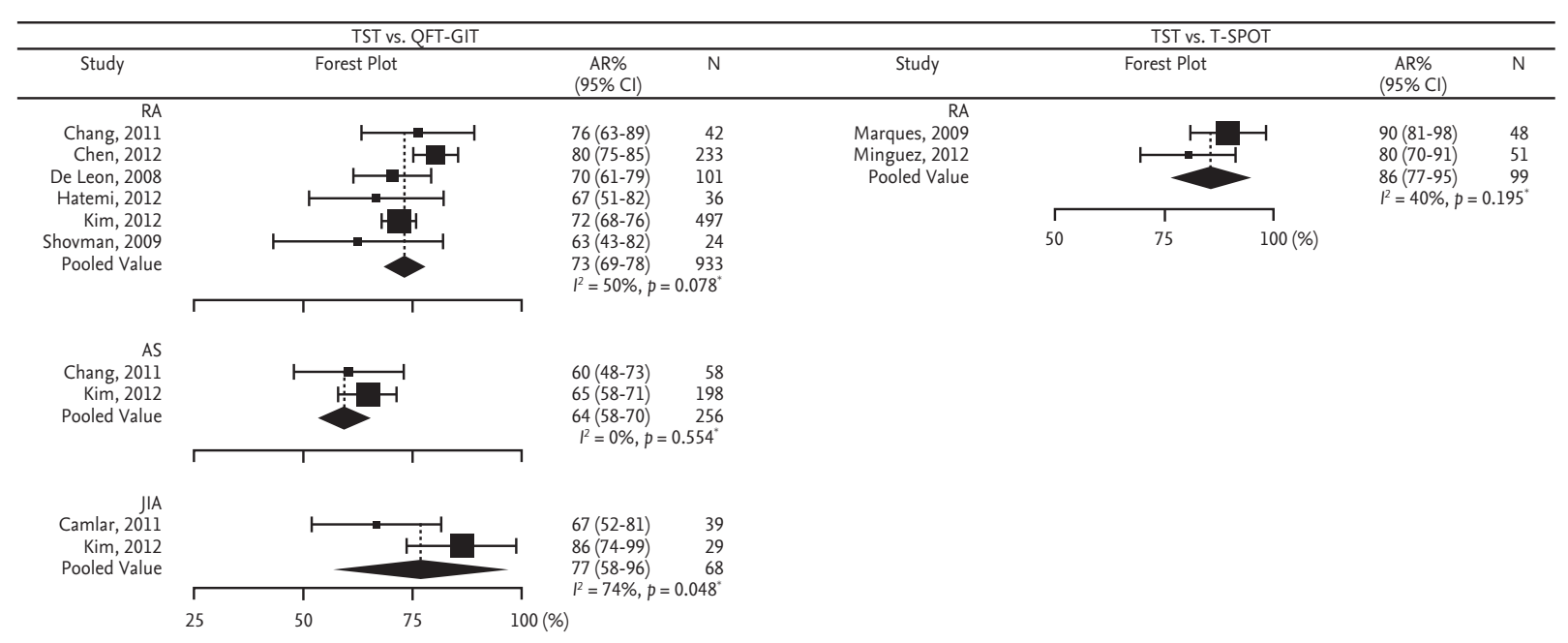

B

* $p$-value of Q-test.

Figure 4. Pooled analysis of the agreement percentage between the TST and interferon-gamma release assay (IGRA) tests (A) by tuberculosis endemic area and (B) by disease type. TST, tuberculin skin test; QFT-GIT, QuantiFERON-TB Gold In Tube; TSPOT, T-SPOT.TB test; AR, agreement rate; CI, confidence interval; N, number; RA, rheumatoid arthritis; AS, ankylosing spondylitis; JIA, juvenile idiopathic arthritis.

pecially in areas with an intermediate TB burden, such as Korea. It has also been shown that the risk of TST conversion increases significantly over 3 years of therapy [30]. Some differences in recommendations due to endemic prevalence might be reasonable, but its effectiveness has yet to be proven. In our study, $55.5 \%$ of patients were targeted to treat LTBI according to the either TST or QFT positive strategy in the high endemic regions. LTBI treatment for more than half of patients before starting with biologic agents has the distinct possibility of increasing the occurrence of adverse events such as liver function abnormalities and decreasing compliance related to polypharmacy [31].

Our study suggested that the results of both tests differ 

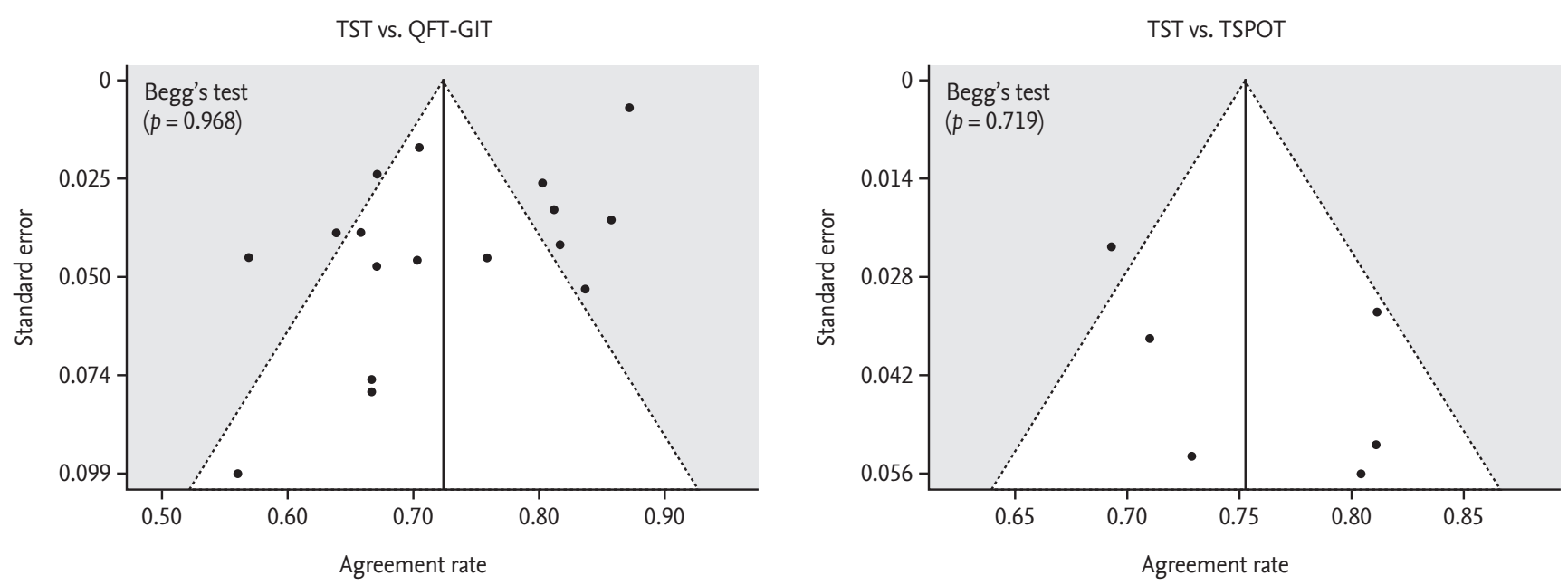

Figure 5. Assessment of publication bias through funnel plots for the agreement percentage. (A) Tuberculin skin test (TST) vs. QuantiFERON TB-Gold In-Tube test (QFT-GIT, Cellestis Ltd.) and (B) TST vs. T-SPOT.TB (TSPOT, Oxford Immunotec Inc.).

according to the underlying rheumatic disease and the endemic TB origins of the patient. Therefore, an optimal screening strategy for LTBI is needed that considers both tuberculosis risk and patient disease. Considering the different false positivity of screening methods and the adverse drug reactions due to unnecessary prophylactic medications, the selection of a screening strategy requires circumspection. Thus, balancing cost, efficacy, and safety should be considered in choosing a screening strategy to determine the prophylactic treatments to be employed.

Our study had several limitations. The most important limitation might be the wide heterogeneity of the summarized results. Diagnostic results came from patients with all rheumatic diseases, of various statuses relating to BCG vaccination and corticosteroid use; they included a minor population of TNF inhibitor use, and different country settings, all of which could contribute to the inconsistencies. Furthermore, a thorough subgroup analysis by underlying disease was impossible since many articles report their diagnostic results only for aggregated patients with rheumatic diseases, rather than separately for each type of disease.

In conclusion, a lower level of agreement between the TST and IGRAs was found in the low-to-moderate endemic countries than in the high endemic countries, and in AS populations as compared to the other rheumatic disease populations. Thus, differing strategies may be needed for patients with rheumatic diseases when detecting LTBI in clinical settings so that endemic origins and underlying diseases are also taken into consideration.

\section{KEY MESSAGE}

1. The latent tuberculosis infection (LTBI) screening test results by tuberculin skin test and interferon-gamma release assay among rheumatic patients were inconsistent, which were also modified by endemic status of origin country and underlying disease.

2. Our study suggest a different clinical strategy may be required to detect LTBI by considering patient's origin of country and precise nature of underlying disease.

\section{Conflict of interest}

No potential conflict of interest relevant to this article was reported.

\section{REFERENCES}

1. Bouza E, Moya JG, Munoz P. Infections in systemic lupus erythematosus and rheumatoid arthritis. Infect Dis Clin North Am 2001;15:335-361. 
2. Ledingham J, Wilkinson C, Deighton C. British Thoracic Society (BTS) recommendations for assessing risk and managing tuberculosis in patients due to start anti-TNF-\{alpha\} treatments. Rheumatology (Oxford) 2005;44:1205-1206.

3. Mariette X, Salmon D. French guidelines for diagnosis and treating latent and active tuberculosis in patients with RA treated with TNF blockers. Ann Rheum Dis 2003;62:791.

4. Singh JA, Furst DE, Bharat A, et al. 2012 Update of the 2008 American College of Rheumatology recommendations for the use of disease-modifying antirheumatic drugs and biologic agents in the treatment of rheumatoid arthritis. Arthritis Care Res (Hoboken) 2012;64:625639.

5. Camlar SA, Makay B, Appak O, et al. Performance of tuberculin skin test and interferon gamma assay for the diagnosis of latent tuberculosis infection in juvenile idiopathic arthritis. Clin Rheumatol 2011;30:1189-1193.

6. Chang B, Park HY, Jeon K, et al. Interferon- $\gamma$ release assay in the diagnosis of latent tuberculosis infection in arthritis patients treated with tumor necrosis factor antagonists in Korea. Clin Rheumatol 2011;30:1535-1541.

7. Chen DY, Shen GH, Chen YM, Chen HH, Hsieh CW, Lan JL. Biphasic emergence of active tuberculosis in rheumatoid arthritis patients receiving TNF $\alpha$ inhibitors: the utility of IFN $\gamma$ assay. Ann Rheum Dis 2012;71:231-237.

8. Ponce de Leon D, Acevedo-Vasquez E, Alvizuri S, et al. Comparison of an interferon-gamma assay with tuberculin skin testing for detection of tuberculosis (TB) infection in patients with rheumatoid arthritis in a TBendemic population. J Rheumatol 2008;35:776-781.

9. Hatemi G, Melikoglu M, Ozbakir F, Tascilar K, Yazici H. QuantiFERON-TB Gold in tube assay for the screening of tuberculosis before and during treatment with tumor necrosis factor alpha antagonists. Arthritis Res Ther 2012;14:R147.

10. Hsia EC, Schluger N, Cush JJ, et al. Interferon- $\gamma$ release assay versus tuberculin skin test prior to treatment with golimumab, a human anti-tumor necrosis factor antibody, in patients with rheumatoid arthritis, psoriatic arthritis, or ankylosing spondylitis. Arthritis Rheum 2012;64:2068-2077.

11. Klein M, Jarosova K, Forejtova S, et al. QuantiFERON TB Gold and tuberculin skin tests for the detection of latent tuberculosis infection in patients treated with tumour necrosis factor alpha blocking agents. Clin Exp Rheumatol 2013;31:111-117.

12. Kim JH, Cho SK, Han M, et al. Factors influencing discrepancies between the QuantiFERON-TB gold in tube test and the tuberculin skin test in Korean patients with rheumatic diseases. Semin Arthritis Rheum 2013;42:424432.

13. Marques CD, Duarte AL, de Lorena VM, et al. Evaluation of an interferon gamma assay in the diagnosis of latent tuberculosis infection in patients with rheumatoid arthritis. Rheumatol Int 2009;30:57-62.

14. Mariette X, Baron G, Tubach F, et al. Influence of replacing tuberculin skin test with ex vivo interferon $\gamma$ release assays on decision to administer prophylactic antituberculosis antibiotics before anti-TNF therapy. Ann Rheum Dis 2012;71:1783-1790.

15. Martin J, Walsh C, Gibbs A, et al. Comparison of interferon \{gamma\} release assays and conventional screening tests before tumour necrosis factor \{alpha\} blockade in patients with inflammatory arthritis. Ann Rheum Dis 2010;69:181-185.

16. Minguez S, Latorre I, Mateo L, et al. Interferon-gamma release assays in the detection of latent tuberculosis infection in patients with inflammatory arthritis scheduled for anti-tumour necrosis factor treatment. Clin Rheumatol 2012;31:785-794.

17. Paluch-Oles J, Magrys A, Koziol-Montewka M, Koszarny A, Majdan M. Identification of latent tuberculosis infection in rheumatic patients under consideration for treatment with anti-TNF- $\alpha$ agents. Arch Med Sci 2013;9:112-117.

18. Ringrose JS, Sanche SE, Taylor-Gjevre RM. Detecting latent tuberculosis infection during anti-tumor necrosis factor therapy. Clin Exp Rheumatol 2011;29:790-794.

19. Saidenberg-Kermanac'h N, Semerano L, Naccache JM, et al. Screening for latent tuberculosis in anti-TNF- $\alpha$ candidate patients in a high tuberculosis incidence setting. Int J Tuberc Lung Dis 2012;16:1307-1314.

20. Scrivo R, Sauzullo I, Mengoni F, et al. Serial interferon- $\gamma$ release assays for screening and monitoring of tuberculosis infection during treatment with biologic agents. Clin Rheumatol 2012;31:1567-1575.

21. Shovman O, Anouk M, Vinnitsky N, et al. QuantiFERON-TB Gold in the identification of latent tuberculosis infection in rheumatoid arthritis: a pilot study. Int J Tuberc Lung Dis 2009;13:1427-1432.

22. Vassilopoulos D, Stamoulis N, Hadziyannis E, Archiman- 
dritis AJ. Usefulness of enzyme-linked immunospot assay (Elispot) compared to tuberculin skin testing for latent tuberculosis screening in rheumatic patients scheduled for anti-tumor necrosis factor treatment. J Rheumatol 2008; 35:1271-1276.

23. Vassilopoulos D, Tsikrika S, Hatzara C, et al. Comparison of two gamma interferon release assays and tuberculin skin testing for tuberculosis screening in a cohort of patients with rheumatic diseases starting anti-tumor necrosis factor therapy. Clin Vaccine Immunol 2011;18:21022108.

24. Xie X, Chen JW, Li F, Tian J, Gao JS, Zhang D. A T-cellbased enzyme-linked immunospot assay for tuberculosis screening in Chinese patients with rheumatic diseases receiving infliximab therapy. Clin Exp Med 2011;11:155161.

25. Ruan Q, Zhang S, Ai J, Shao L, Zhang W. Screening of latent tuberculosis infection by interferon- $\gamma$ release assays in rheumatic patients: a systemic review and meta-analysis. Clin Rheumatol 2016;35:417-425.

26. Pouchot J, Grasland A, Collet C, Coste J, Esdaile JM, Vinceneux P. Reliability of tuberculin skin test measurement.
Ann Intern Med 1997;126:210-214.

27. Menzies D. Interpretation of repeated tuberculin tests: boosting, conversion, and reversion. Am J Respir Crit Care Med 1999;159:15-21.

28. Iannone F, Cantini F, Lapadula G. Diagnosis of latent tuberculosis and prevention of reactivation in rheumatic patients receiving biologic therapy: international recommendations. J Rheumatol Suppl 2014;91:41-46.

29. Beglinger C, Dudler J, Mottet C, et al. Screening for tuberculosis infection before the initiation of an anti-TNF-alpha therapy. Swiss Med Wkly 2007;137:620-622.

30. Park JH, Seo GY, Lee JS, Kim TH, Yoo DH. Positive conversion of tuberculin skin test and performance of interferon release assay to detect hidden tuberculosis infection during anti-tumor necrosis factor agent trial. J Rheumatol 2009;36:2158-2163.

31. Vanhoof J, Landewe S, Van Wijngaerden E, Geusens P. High incidence of hepatotoxicity of isoniazid treatment for tuberculosis chemoprophylaxis in patients with rheumatoid arthritis treated with methotrexate or sulfasalazine and anti-tumour necrosis factor inhibitors. Ann Rheum Dis 2003;62:1241-1242. 


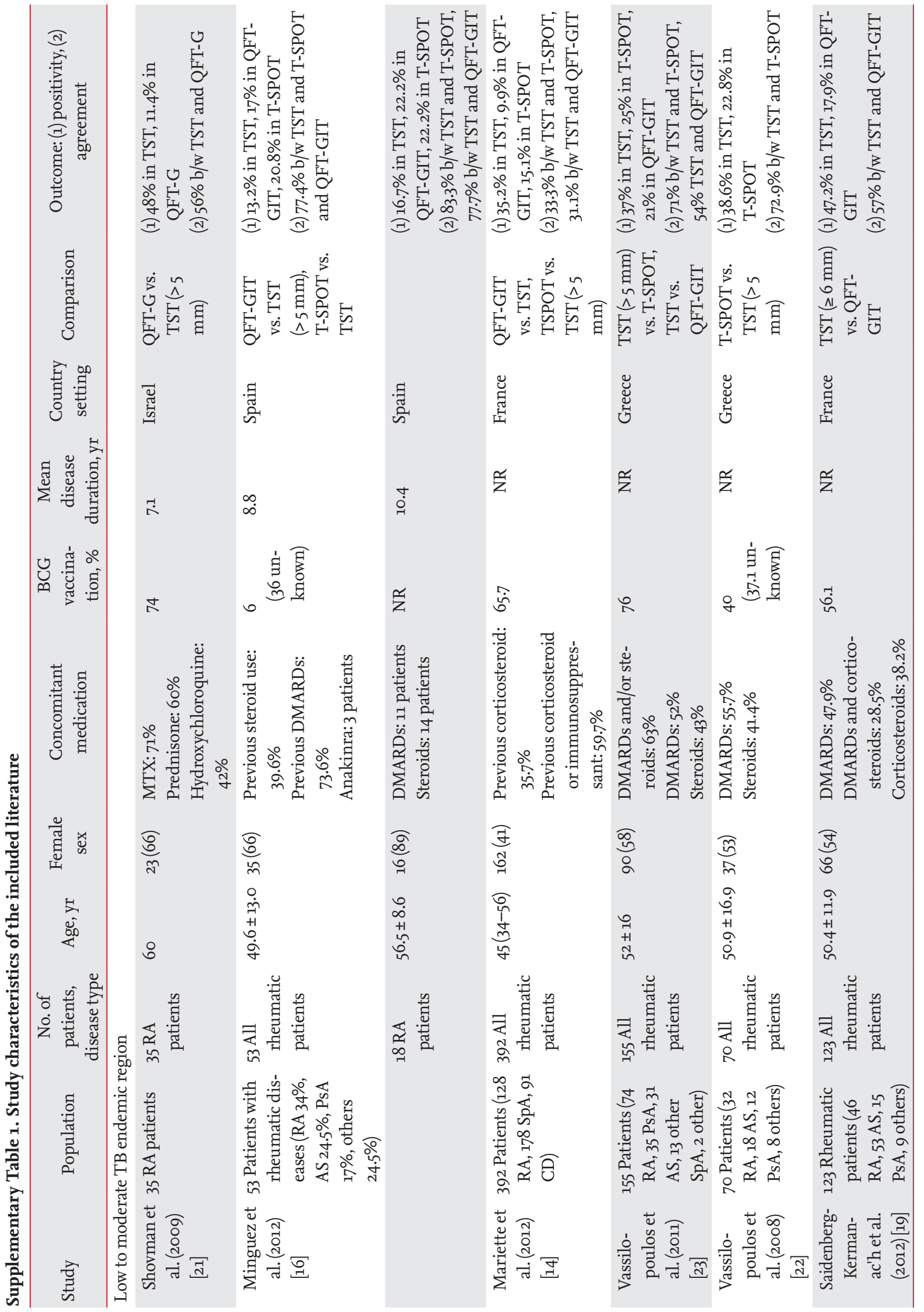




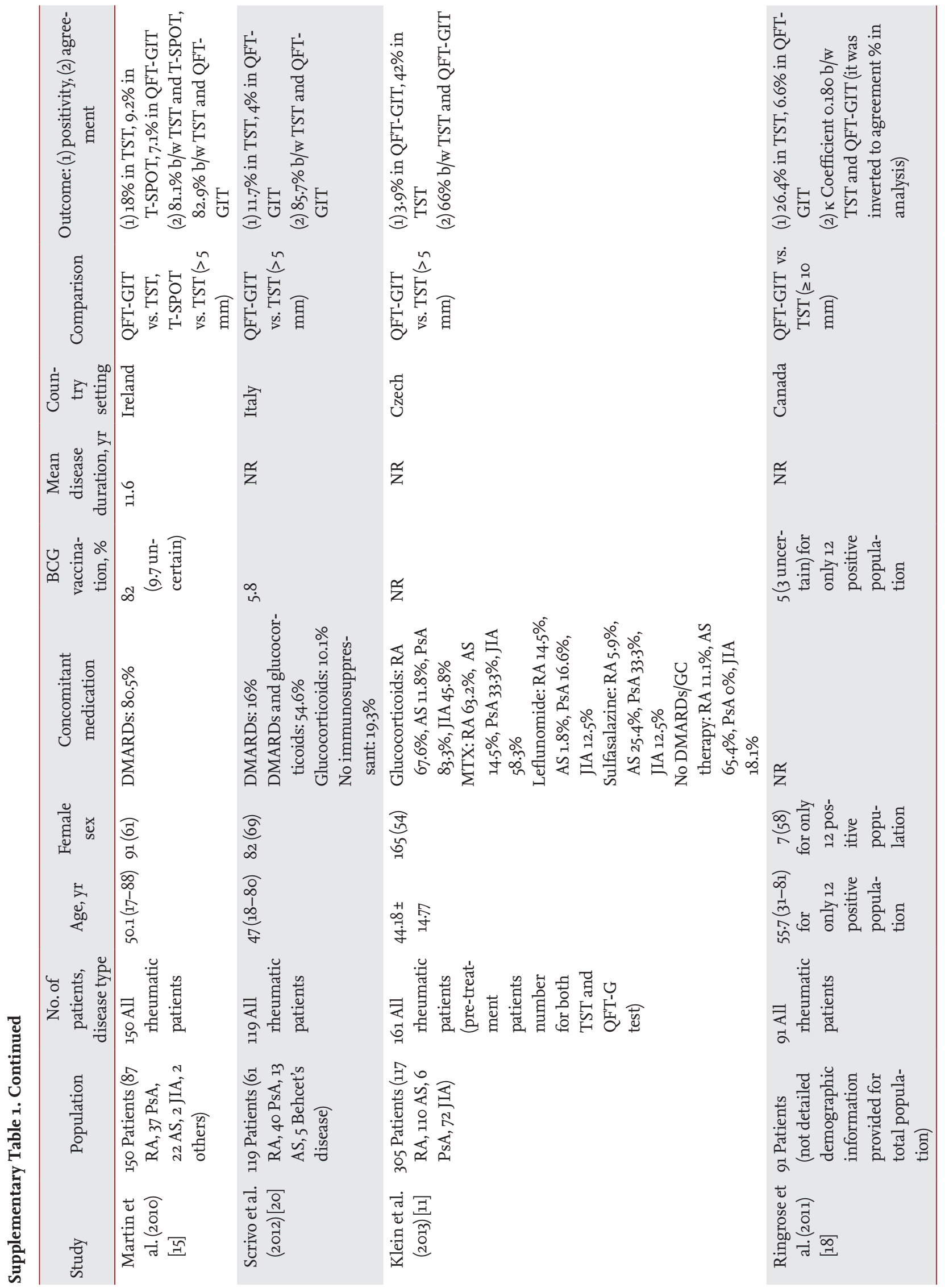




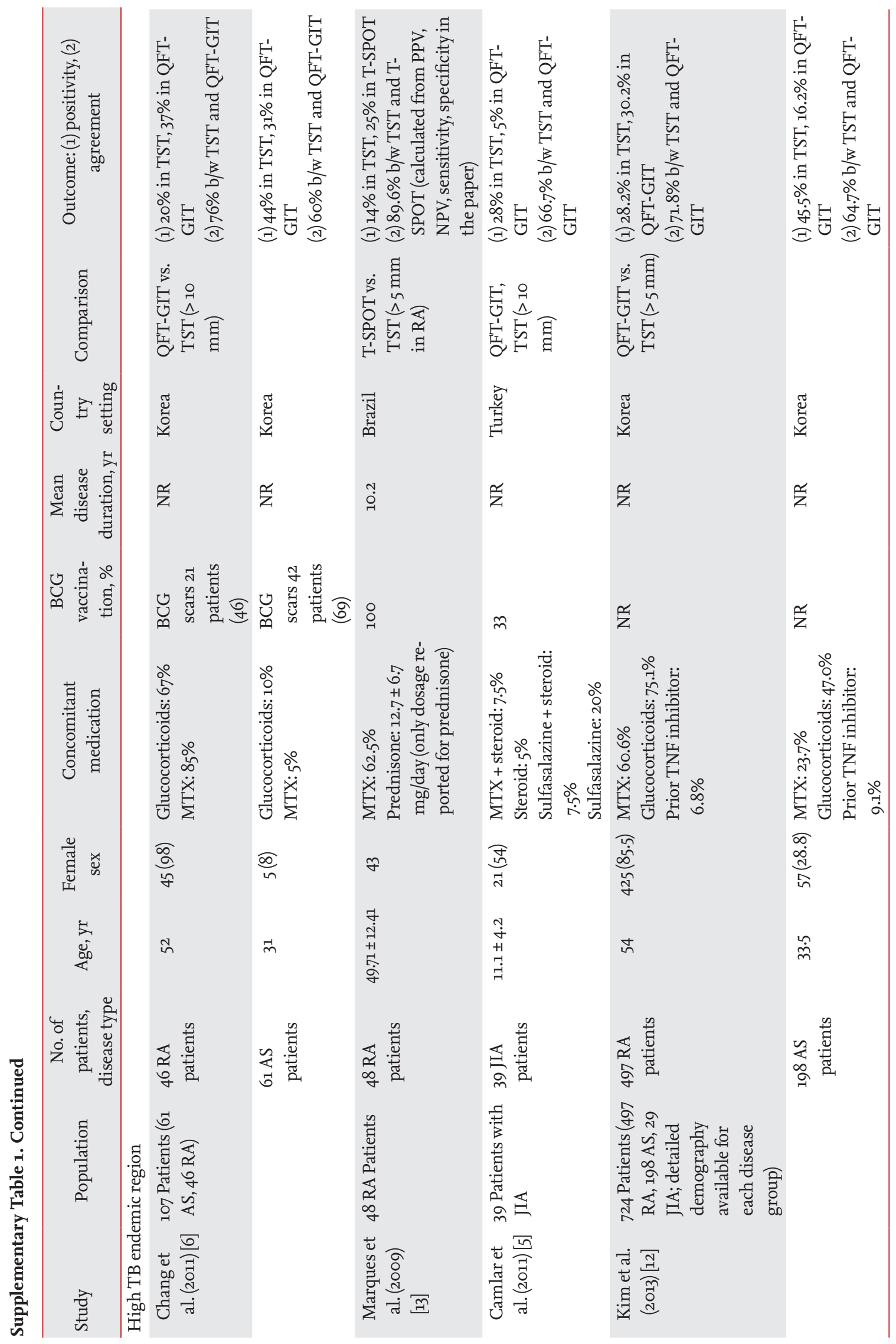




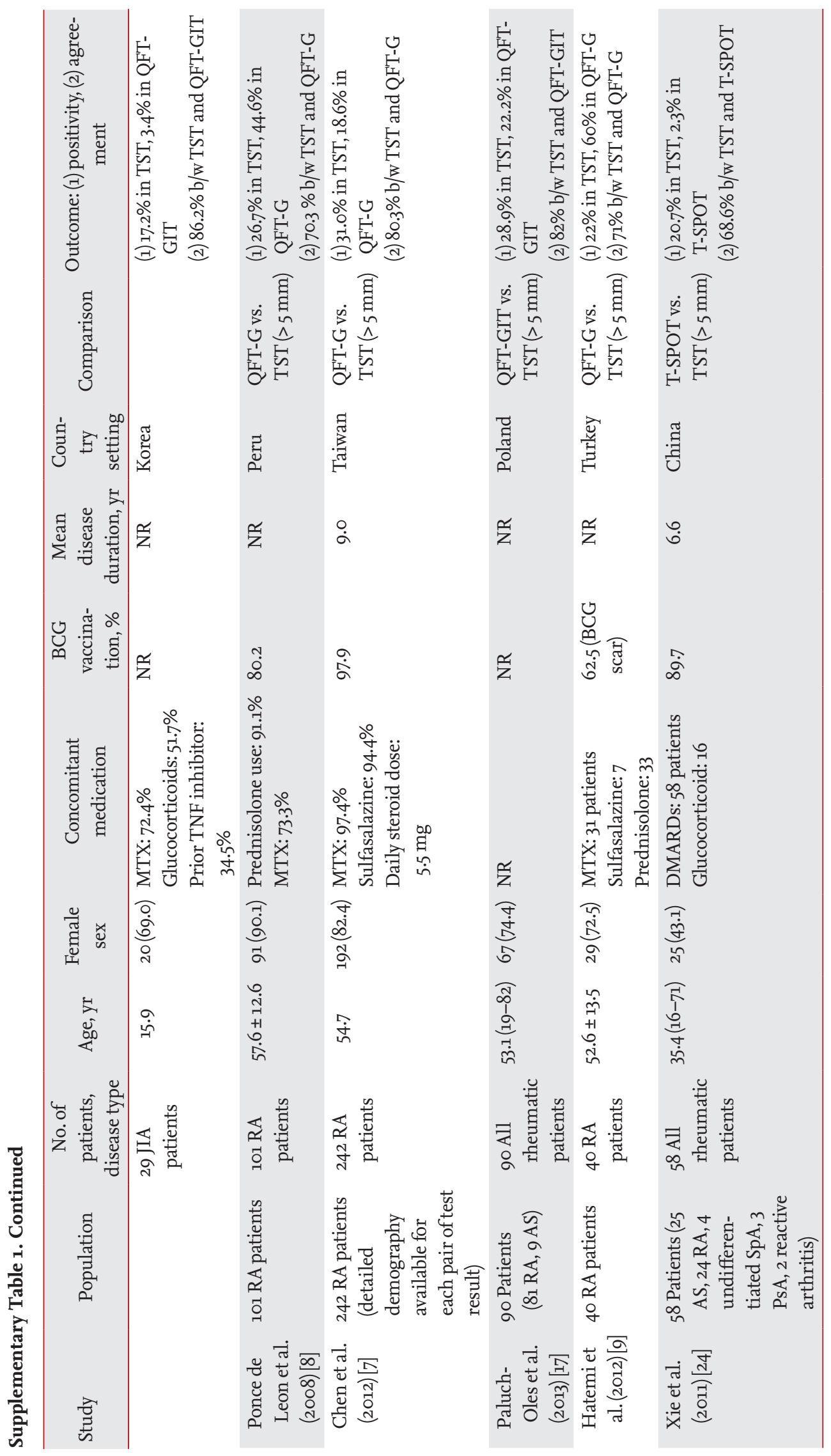




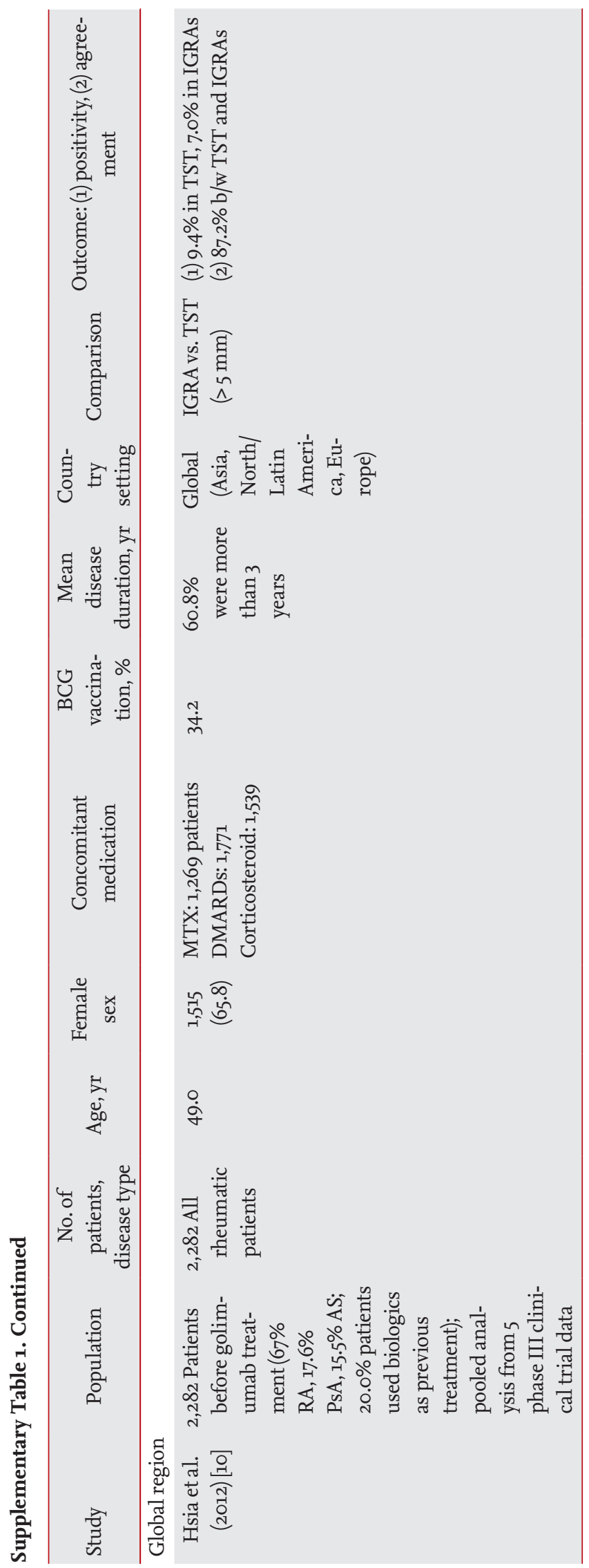

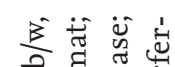

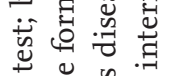

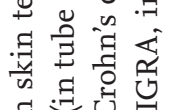

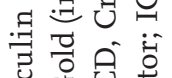

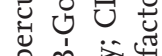

卧焉

的兄荢员

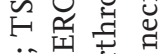

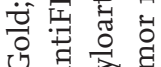

규 졸

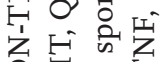

O

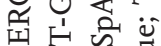

留望

表昆范

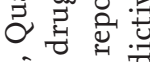

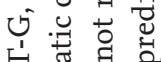

劉总

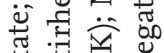

实

范㻤完

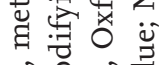

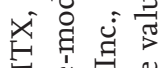

$\sum$ 虫

:

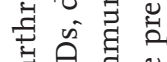

. त्वे 突

ब.

त्ञ

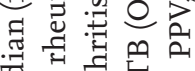

还焉

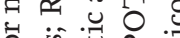

\%.

की 0 H

+ 䓀

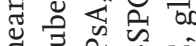

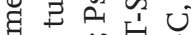

के

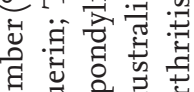

娄

范

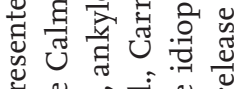

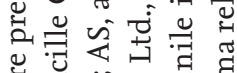

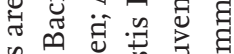

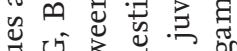

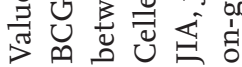

